\title{
Remaining Useful Life Prediction of Quay Crane Hoist Gearbox Bearing under Dynamic Operating Conditions Based on ARIMA-CAPF Framework
}

\author{
Zhiwei Sun $(\mathbb{D}$, Xiong Hu $(\mathbb{D}$, and Kai Dong $(\mathbb{D}$ \\ Logistic Engineering College, Shanghai Maritime University, Shanghai 201306, China \\ Correspondence should be addressed to Xiong Hu; huxiong@shmtu.edu.cn
}

Received 12 August 2021; Revised 3 November 2021; Accepted 2 December 2021; Published 21 December 2021

Academic Editor: Tony Murmu

Copyright (c) 2021 Zhiwei Sun et al. This is an open access article distributed under the Creative Commons Attribution License, which permits unrestricted use, distribution, and reproduction in any medium, provided the original work is properly cited.

\begin{abstract}
The remaining useful life (RUL) prediction of quay crane (QC) bearings is of great significance to port production safety. An RUL prediction framework of QC bearing under dynamic conditions is proposed. Firstly, the load is discretized, and the corresponding operating conditions are classified. Then, the Autoregressive Integrated Moving Average (ARIMA) model is utilized to predict the load and corresponding operating conditions. Secondly, a Wiener process considering degradation rates and jump coefficients under different operating conditions is developed as the state transfer function. Finally, a condition-activated particle filter (CAPF) is proposed to predict the system state and the bearing's RUL. The proposed prediction framework is verified by the hoist bearing life cycle data from a port in Shanghai collected by the NetCMAS system. The prediction results by the ARIMA-CAPF framework in comparison with three other prediction strategies identify the effectiveness.
\end{abstract}

\section{Introduction}

As special equipment, QC is the most frequently used equipment in the area of port container transportation. The health condition of the hoist gearbox determines the working efficiency and production safety of QCs. As the crucial components of the hoist system, bearings will withstand the vibration shock caused by the strong dynamic load in the periodical process of the containers' lifting and descending. In the long term, it will lead to bearings' performance degradation or even sudden failure, resulting in downtime and even casualties $[1,2]$. Condition monitoring and life prediction of bearings can avoid the unnecessary shutdown and improve the reliability and safety of QC bearings $[3,4]$.

In some papers, the relationships between the bearings' degradation and the evolution of vibration, temperature, and other types of signals under unchanged operating conditions have been studied. These researches can be divided into two main categories: model-based methods and data-driven methods [5]. Generally, the model-driven methods establish a physical or mathematical model for bearings' RUL prediction. Lei et al. [6] established a bearing degradation model based on the Paris-Erdogan model and predicted RUL with a particle filtering algorithm. Liao et al. [7] employed a proportional hazards model along with a logistic regression model to predict the RUL of bearings. Qian et al. [8] used recurrence quantification analysis (RQA) to generate degradation states and combined autoregression (AR) model with the Kalman filter (KF) to predict bearings' RUL. On the other hand, data-driven methods focus more on collected data for RUL prediction when the failure mechanism is too complex. Aye et al. [9] predicted the degradation trends of rolling element bearings using an integrated GPR model. Wang et al. [10] used multiple deep autoencoder models to extract linear reliability indicators for RUL prediction under certain load and speed conditions. Ali et al. [11] utilized Simplified Fuzzy Adaptive Resonance Theory Map (SFAM) neural network as a degradation model and proposed a smoothing phase to find the optimal RUL prediction. These two kinds of methods both have acquired accurate RUL prediction results. But these pieces of literature assume that 
the main operating conditions of the system remain unchanged throughout its life cycle. Unfortunately, excluding the influences of operational conditions out of degradation modeling is not always realistic and appropriate. The variation of conditions may cause changes in signal amplitudes and degradation rates and then affect the RUL prediction. For example, the increase of load will cause a higher vibration level, simultaneously accelerating the degradation process of bearing. Hence, it is of vital importance to take the changing operating conditions into consideration when making RUL prediction.

Recently, some papers have considered how the dynamic operating conditions influence the RUL prediction. The impacts of operating condition change on vibration signal can be summarized as two parts [12]: (1) changes of degradation rates in different operating conditions and (2) signal jumps when the operating conditions switch. Liao et al. [13] estimated the degradation rates by Bayesian framework when the operating conditions were piecewise constant, and the RUL was predicted by the Monte Carlo simulation. Zhao et al. [14] used the Paris-Erdogan model to describe the crack growth of spur gear. The degradation rates were adjusted according to the dynamic load, and the RUL is predicted combined with finite element analysis. Peng et al. [15] compared the constant, monotone, and S-type degradation rates and predicted the RUL with the inverse Gaussian process. Pradeep et al. [16] took load, speed, temperature, humidity, and other factors as degradation parameters and predicted the bearing life by using the Weibull degradation model. Xu et al. [17] used a two-step unbiased estimation method to estimate degradation rates, and the Wiener process is adopted to predict the RUL. Li et al. [18] introduced signal jumps as two jump coefficients along with degradation rates into the two-factor state-space (F2S2) model and gave the closed-form solution of residual life distribution under time-varying operating conditions.

These pieces of literature studied bearings' RUL prediction under dynamical operating conditions, but all assume that the future operating conditions of the same bearing are predetermined and piecewise constant but ignored the fact that operating conditions could change dynamically and cannot be predetermined during system deterioration. This phenomenon is consistent with the actual operating scenario, and therefore, it is necessary to predict the load in order to determine the corresponding operating condition before RUL prediction. At present, the main challenge is that the prediction of operating conditions in bearing RUL prediction has not been considered.

In order to study the impacts of dynamic conditions on RUL prediction, we try to establish the life prediction model under no-predetermined dynamic operating conditions. We add load prediction and operating condition classification steps before life prediction and put forward the updating method of parameters changing with operating conditions in the prediction process, which has not been considered comprehensively before. On the basis of this, the method proposed in this paper divides the RUL prediction procedures of bearing into two parts: first, the load is predicted, and different operating conditions are classified by load discretization result. Second, the system state is updated, and the RUL of the bearing is predicted based on the prediction results of load and corresponding operating conditions.

Specifically, the equidistant discretization method is adopted to classify the load and determine different operating conditions, and the ARIMA model is utilized to predict the load. Then, a developed Wiener process is adopted as the state transition function to describe the degradation process of the system state. The particle filter algorithm improved by a condition activation vector is applied to predict the system state and update the degradation rates and signal jump coefficients so as to realize the RUL prediction.

The main contributions of this work are as follows:

(1) A framework of RUL prediction based on the ARIMA model and CAPF method under off-design operating conditions is proposed.

(2) A system state prediction method based on PF under dynamic conditions is improved.

(3) The impacts of the jump coefficients and degradation rates under dynamic operating conditions are discussed.

The remainder of this paper is summarized as follows.

In Section 2, the involved background theories are briefly introduced. In Section 3, the procedure of the RUL prediction framework is proposed. In Section 4, the effectiveness of the proposed method is verified, and the results are discussed. Finally, the conclusion of this paper is given in Section 5 .

\section{Theory Background}

2.1. ARIMA Model. Load prediction can be regarded as the prediction of time series. The time series model can express the change of time series by extrapolation mechanism without considering the influence of other variables on the target value. ARIMA model is one of the time-domain analysis methods dealing with time series [19]. It is developed based on the autoregressive (AR) model and moving average (MA) model. The ARIMA model stabilizes nonstationary time series by a difference process, which can overcome the disadvantage that the AR model and MA model can only deal with stationary time series. At the same time, compared with the autoregressive conditional heteroskedasticity (ARCH) model and its derived models, the ARIMA model has a simpler structure and fewer input variables [20]. It has been widely used in short-term [21-23] and mid- and long-term load prediction [24-26]. ARIMA model is formulated as ARIMA $(P, D, Q)$, where $P, D$, are AR model order, differential order, and MA model order, respectively. The general expression of the ARIMA model is defined as follows:

$$
Z_{t}=\mu+\sum_{i=1}^{P} \alpha_{i} Z_{t-i}+\sum_{j=1}^{Q} \beta_{j} \varepsilon_{t-j},
$$

where $Z_{t}$ is stationary series after differential operation and $\mu$ is the constant term and represents the mean of the stochastic time series. $\alpha_{i}$ and $\beta_{j}$ are AR and MA model 
parameters, respectively. $\varepsilon_{t-j}$ is the error between the predicted value and the actual value at time $t-j$.

2.2. Particle Filter. The PF is a filtering method based on the Bayesian estimation theory and Monte Carlo method. A set of random particles are adopted to describe the state of the system. The PF has been widely used for bearing RUL prediction in both bearings $[10,27]$ and lithium-ion batteries [28-30] under unchanged operating conditions since it applies to any form of state-space model (linear or nonlinear; Gaussian or non-Gaussian) [31]. Consider a dynamic system model as follows:

$$
\begin{aligned}
& \theta_{t}=f\left(\theta_{t-\Delta t}, \varphi_{t-\Delta t}\right), \\
& x_{t}=h\left(\theta_{t}, \omega_{t}\right),
\end{aligned}
$$

where $f(\cdot)$ and $h(\cdot)$ are the state transition and the measurement functions, respectively. $\varphi_{t-\triangle t}$ and $\omega_{t}$ are the corresponding noises. $x_{t}$ is the system state at time $t$.

The system model can be developed based on some datadriven empirical models, such as Wiener process models $[32,33]$ and gamma process models $[34,35]$. In this paper, we apply a common Wiener process model as the basis degradation function of our modeling framework, and we will introduce it in the next section.

Suppose that the prior probability density function of initial state $x_{0}$ is $p\left(x_{0}\right)$. The main procedure of system state estimation through $\mathrm{PF}$ is presented as follows:

(1) Initialization: generate $N$ particles $x_{t}^{i}$ from the prior distribution $p\left(x_{0}\right)$ and $N$ corresponding weights $\omega_{t}^{i}$, $i=1,2, \ldots, N$.

(2) Prediction: predict the prior probability of the current state $x_{t}$ by utilizing equation (1).

(3) Update: calculate and normalize the weights of particles by

$$
\begin{aligned}
& \widetilde{\omega}_{t}^{i}=\frac{\omega_{t-\Delta t}^{i}}{\sqrt{2 \pi \widetilde{\sigma}^{2}}} \exp \left(-\frac{\left(y_{t}-x_{t}^{i}\right)^{2}}{2 \widetilde{\sigma}^{2}}\right), \\
& \widetilde{\omega}_{t}^{i}=\frac{\widetilde{\omega}_{t}^{i}}{\sum_{i=1}^{N} \widetilde{\omega}_{t}^{i}}, \\
& \theta_{t}=\sum_{i=1}^{N} \widetilde{\omega}_{t}^{i} \theta_{t}^{i},
\end{aligned}
$$

where $\omega_{t}^{i}$ is the weight of $i$ th particle at time $t$. $q\left(x_{t}^{i} \mid x_{t-\Delta t}^{i}, y_{t}\right)$ is the importance density function and $p\left(y_{t} \mid x_{t}^{i}\right)$ and $p\left(x_{t}^{i} \mid x_{t-\triangle t}^{i}\right)$ are the likelihood function and the state transition probability density function.

(4) Resampling: remove particles with small weight and copy particles with large weights. The weights of resampled particles are reset to $\omega_{t}^{i}=1 / N$.
2.3. RUL Prediction. The RUL is defined as the time interval from the current time to the end of the useful life [36], which is expressed as

$$
r_{k}=t_{E o L}-t_{k}
$$

where $t_{\mathrm{EoL}}$ is the end of the useful life, $t_{k}$ is the current time, and $r_{k}$ is the RUL at $t_{k}$. For a PF model, the RUL can be defined as the time interval between the current time and the time when the system state reaches the failure threshold, which is expressed as

$$
r_{k}=\inf \left(l: x\left(l+t_{k}\right) \geq \gamma\right),
$$

where $x\left(l+t_{k}\right)$ is the system state at $l+t_{k}$, inf(.) represents the inferior limit of a variable, and $\gamma$ is the failure threshold.

\section{RUL Prediction Method Based on ARIMA and CAPF}

In this section, we will elaborate on our ARIMA-CAPF RUL prediction framework. This framework mainly includes three steps: (1) operating condition initialization, (2) realtime prediction and update, and (3) degradation assessment and RUL prediction. The ARIMA-CAPF RUL prediction flowchart is shown in Figure 1. Each step is described in detail in the following subsections.

3.1. State Equation Development. The operating condition parameters are mainly divided into two parts: the state jumps when the operating condition changes and the degradation rates assigned to each operating condition. The first order linear Wiener process is considered as

$$
W(t)=W(0)+\eta t+\sigma_{B} B(t)
$$

where $W(t)$ represents the state of the system at time $t$ and $W(0)$ represents the initial state value of the system. It is determined by the initial system quality and usually set as 0 . $\eta$ is the degradation rate (assumed to be a constant under fixed operating conditions but will change with the changes of operating conditions), $B(t)$ is the standard Brownian motion, and $\sigma_{B}$ is the diffusion term. Based on the consideration of the dynamic operating conditions, we establish the following degradation equations:

$$
x(t)=a_{o_{t-\Delta t}, o_{t}}\left(x(t-\Delta t)+\eta_{o_{t}} \Delta S+b_{o_{t-\Delta t}, o_{t}}\right)+\omega_{t},
$$

where $x(t)$ represents the state of the system at time $t, \Delta t$ represents the time interval, and $o_{t-\Delta t}$ and $o_{t}$ represent the operating conditions at time $t-\Delta t$ and time $t$, respectively. For example, if there are three different conditions, $o_{t}$ will take values from the set $\{1,2,3\} . a_{o_{t-\Delta t}, o_{t}}$ and $b_{o_{t-\Delta t}, o_{t}}$ are two signal jump coefficients when the operating condition transfers from $o_{t-\Delta t}$ to $o_{t}$, and these two variables together with $\eta_{o_{t}}$ represent the effects of load level on degradation. $\eta_{o_{t}}$ reflects the signal transfer direction. It could be positive or negative. For example, if the operating condition transfers 


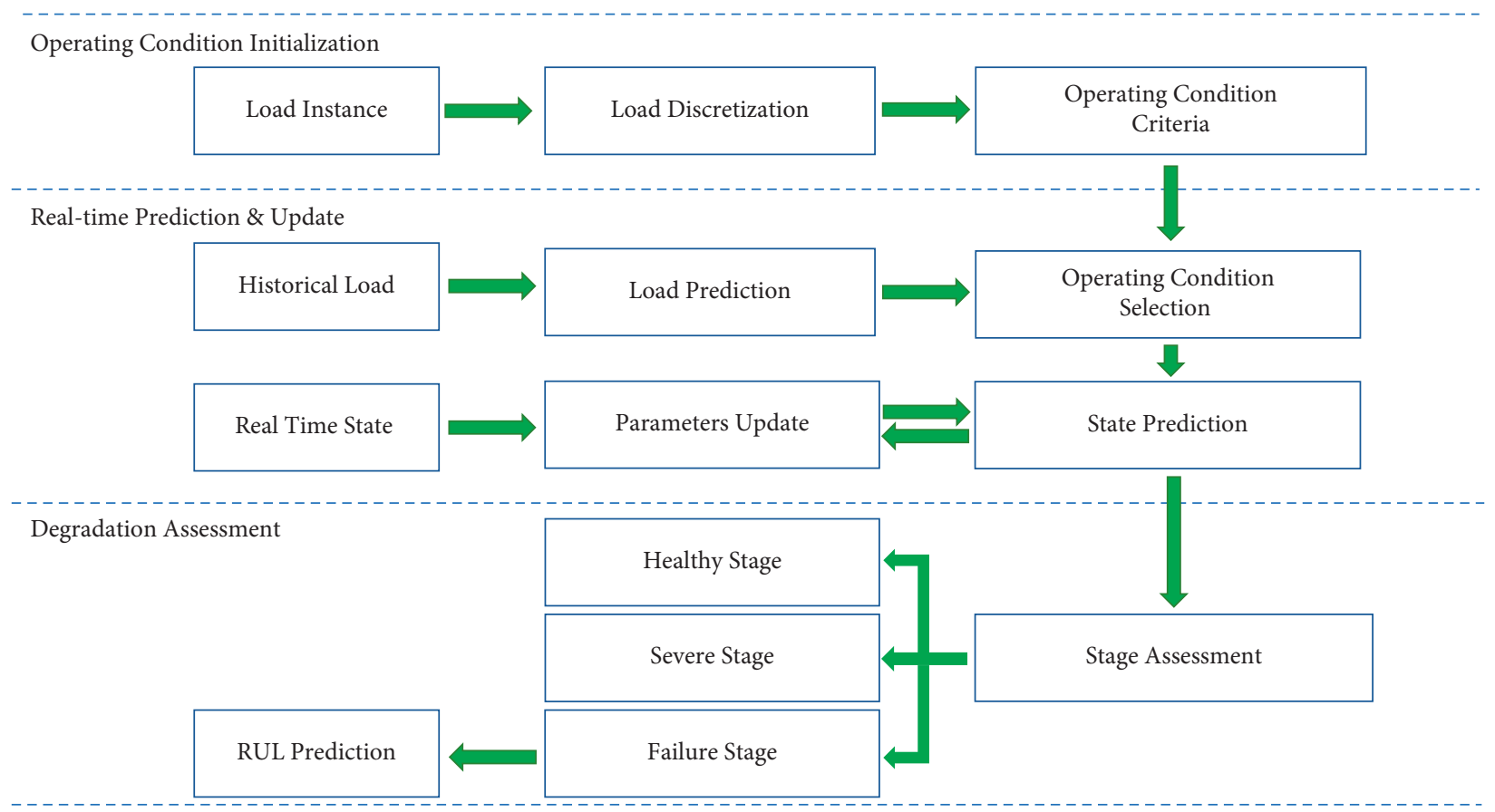

FIGURE 1: Flowchart of ARIMA-CAPF RUL prediction framework under dynamic operating conditions.

from a high load level to a lower one, $\eta_{o_{t}}$ could be negative. If the operating condition remains unchanged, then $\eta_{o_{t}}$ will be set as $1 . \omega_{t}$ is the measurement noise at time $t$. It represents the measurement error caused by the noise from sensors or working environment, which can be obtained by long-term statistics of the data measured by the sensors. $\triangle S$ denotes the load increment from time $t-\Delta t$ to time $t$.

\subsection{Load Discretization and Operating Condition} Initialization. As special equipment, each QC has its unique mechanical characteristics, so it is difficult to obtain prior degradation information such as degradation rate from the historical data of other QCs. Generally, the degradation process of the bearing of the hoist gearbox can be divided into three stages: stable stage, fault stage, and failure stage [37]. In the stable stage, the system state is relatively stable with slight change under corresponding operating conditions, and the degradation process of the machine in this stage can generally traverse the whole load range. Some researchers have utilized discretization methods to classify the working load [38, 39]. In this paper, an equidistant discretization method is adopted to discretize the load and calculate the operating condition boundary because it is easy to operate and fast to calculate. The specific steps are as follows.

Suppose that there are $k$ operating conditions, the load sequence is $S=\left\{S_{1}, S_{2}, \ldots, S_{m}\right\}$, and $S_{\max }$ and $S_{\min }$ are the maximum and minimum values of the load sequence, respectively; then

(1) calculate the length of each load interval:

$$
\mathrm{LC}=\frac{S_{\max }-S_{\min }}{k} \text {. }
$$

(2) Generate $n$ cut points:

$$
\mathrm{C}=\left\{c_{0}, c_{1}, c_{2}, \ldots, c_{n}\right\},
$$

where $n=k+1, \quad c_{0}=S_{\min }, c_{n}=S_{\max }, \quad c_{i+1}=$ $c_{0}+i * \mathrm{LC}, 1 \leq i \leq n-1$;

(3) Set operating condition criterion:

$$
o_{t}=i, \quad \text { if } c_{i} \leq S_{i} \leq c_{i+1} .
$$

3.3. Real-Time Prediction and Update. The real-time prediction includes two parts: load prediction and system state prediction. We will describe the two parts in the following subsections.

3.3.1. Load Prediction by ARIMA. Before predicting the state of the system, it is necessary to predict the load first. In this paper, we apply the ARIMA model to predict the load with the following steps:

(1) Check the stationarity of the historical load $Z_{t}$. If not, differ $Z_{t}$ until it is stable.

(2) Determine the AR model order $P$ and MA model order $Q$ by using the Box-Jenkins approach [19].

(3) Estimate the ARIMA parameters $\alpha_{i}$ and $\beta_{j}$ by the Akaike information criterion (AIC) [40].

(4) Predict the load $Z_{t+\Delta t}$ by equation (1) at time $t+\Delta t$.

(5) Operating condition initialization: the operating condition $o_{t}$ at time $t$ is determined according to equation (13). 
3.3.2. State Prediction by CAPF. After the load and operating conditions are predicted, the system state can be predicted by PF. In the traditional RUL prediction, the operating condition is assumed to be unchanged, so the degradation parameters are considered to be assigned to only one operating condition. However, when the operating conditions change with time, it is necessary to set different coefficients for each different operating condition and make a selection before state prediction. Therefore, in this paper, we add a condition coefficient selection procedure in the state prediction process by utilizing a proposed CAPF method. The method uses a condition activation vector to select the operating condition and match the corresponding parameters. The specific steps are as follows:

(1) Operating condition factor processing: consider parameters appearing in equation (10)

$$
\begin{aligned}
& \boldsymbol{\eta}_{t}=\left[\eta_{1}, \eta_{2}, \ldots, \eta_{i}, \ldots, \eta_{k}\right]^{T}, \\
& \mathbf{a}_{t}=\left[\begin{array}{c}
a_{11} a_{12} \ldots \ldots a_{1 k} \\
a_{21} a_{21} \ldots \ldots a_{2 k} \\
\ldots \ldots a_{i j} \ldots \ldots . . . \\
a_{k 1} a_{k 2} \ldots \ldots a_{k k}
\end{array}\right], \\
& \mathbf{b}_{t}=\left[\begin{array}{c}
b_{11} b_{12} \ldots \ldots b_{1 k} \\
b_{21} b_{22} \ldots \ldots b_{2 k} \\
\ldots \ldots b_{i j} \ldots \ldots . . . \\
b_{k 1} b_{k 2} \ldots \ldots b_{k k}
\end{array}\right],
\end{aligned}
$$

where $\boldsymbol{\eta}_{t}=\left[\eta_{1}, \eta_{2}, \ldots, \eta_{k}\right]^{T}$ is the degradation rate vector, $\eta_{i}(1 \leq i \leq k)$ represents the degradation rate under ith condition, $\mathbf{a}_{t}$ and $\mathbf{b}_{t}$ are jump coefficient matrices, and $a_{i j}$ and $b_{i j}$ represent two jump coefficients when operating conditions switch from $i$ to $j$.

In order to select an operating condition, we set the activation vector at time $t$ :

$$
\mathbf{V}_{t}=\left[v_{1}, v_{2}, \ldots, v_{i}, \ldots, v_{k}\right] .
$$

Among them,

$$
v_{i}= \begin{cases}1, & \text { if } o_{t}=i, \\ 0, & \text { else. }\end{cases}
$$

Once the operating condition is determined, the degradation rates and jump coefficients at time $t$ can be derived as follows:

$$
\begin{aligned}
\boldsymbol{\eta}_{o_{t}} & =\mathbf{V}_{t} \mathbf{\eta}_{t-\Delta t}, \\
\mathbf{a}_{o_{t-\Delta t}, o_{t}} & =\mathbf{V}_{t-\Delta t} \mathbf{a}_{t-\Delta t} \mathbf{V}_{t}^{T}, \\
\mathbf{b}_{o_{t-\Delta t}, o_{t}} & =\mathbf{V}_{t-\Delta t} \mathbf{b}_{t-\Delta t} \mathbf{V}_{t}^{T} .
\end{aligned}
$$

(2) Particle initialization: let $\boldsymbol{\theta}_{o_{t}}=\left[\eta_{o_{t}}, a_{o_{t-\Delta t}, o_{t}}, b_{o_{t-\Delta t} o_{t}}\right]$ be the parameter vector and generate $N$ particles $\left\{\theta_{0}^{i}\right\}_{i=1: N}$ at the initial time and the corresponding weights $\left\{w_{0}^{i}\right\}_{i=1: N}$.

(3) Predict state: take $\theta_{o_{t}}$ into equation (10) and predict the state.

(4) Updating weights and parameter matrices:

Capture the new state $y_{t}$ at time $t$ and update the particle weights and condition parameters by

$$
\begin{aligned}
& \widetilde{\boldsymbol{\omega}}_{t}^{i}=\frac{\boldsymbol{\omega}_{t-\Delta t}^{i}}{\sqrt{2 \pi \widetilde{\boldsymbol{\sigma}}^{2}}} \exp \left(-\frac{\left(y_{t}-x_{t}^{i}\right)^{2}}{2 \widetilde{\boldsymbol{\sigma}}^{2}}\right), \\
& \widetilde{\boldsymbol{\omega}}_{t}^{i}=\frac{\widetilde{\boldsymbol{\omega}}_{t}^{i}}{\sum_{i=1}^{N} \widetilde{\boldsymbol{\omega}}_{t}^{i}}, \\
& \boldsymbol{\theta}_{o_{t}}=\sum_{i=1}^{N} \widetilde{\boldsymbol{\omega}}_{t}^{i} \theta_{o_{t}}^{i} .
\end{aligned}
$$

(5) Resampling: obtain a new particle set by resampling scheme, and reset all weights to $1 / N$.

3.4. Stage Assessment and RUL Prediction. The state of bearing will not change dramatically in the first two stages, so RUL prediction is only needed in the failure stage. Since the system states would jump dramatically when stages switch under any operating conditions, the $3 \sigma$ principles [41] can be applied to monitor the bearing degradation stage, and the RUL prediction will begin when the bearing degradation reaches the failure stage.

Traditional RUL prediction methods will predict the future states based on the current parameters and operating conditions or assume that the future operating conditions are scheduled profiles. However, most operating conditions in the production process are changing over time. Therefore, before RUL prediction, it is necessary to predict the operating conditions at the future time and estimate the corresponding parameters. The specific steps are as follows:

(1) Set $t=t+\triangle t$, and use the method mentioned in Section 3.3.1 to predict the load $Z_{t}$ and corresponding operating condition $o_{t}$.

(2) Use the method expressed in Section 3.3.2 to predict the system state $x_{t}$ and update the condition parameters assigned to $o_{t}$.

(3) Check whether $x_{t} \geq \gamma$ is true. If yes, $r_{t}=\Delta t$; else, repeat (1) (2) until $x_{t} \geq \gamma$.

\section{Case Study}

4.1. Quay Crane Hoisting Mechanism Gearbox Full-Life LoadState Data. In this section, the validity of the proposed method is verified by the life cycle dataset of a QC hoisting mechanism gearbox obtained from the long-term monitoring by the NetCMAS system. The QC comes from a container terminal in Shanghai. The overview of the QC's structure and relevant measuring points is shown in 
Figure 2. Since the QC was installed, the NetCMAS system has been continuously running for monitoring the mechanical system online for nearly 5 years and 4 months until the bearing, on the high-speed shaft inside the hoisting gearbox, failed with roller abrasion. In this paper, the full-life vibration energy spectrum is utilized as the system state, which is acquired from the accelerometer placed on the surface of the hoist mechanism gearbox, vertical to the highspeed input shaft. A stress measuring point is installed on the boom surface between the inner forestay and outer forestay to capture stress signals. The stress signals recorded the stress variation during the operation and are regarded as the load sets afterward. The above-mentioned measuring points and their placement are shown in Figure 3. The sampling frequency is set as $2500 \mathrm{~Hz}$. Each sampling time is $0.8 \mathrm{~s}$, and the sampling interval is $8 \mathrm{~s}$. The effective values of the collected vibration data and mean values of stress are calculated online to form the online vibration energy spectrum and stress time series.

\subsection{Operating Condition Setting and Degradation Stage Di-} vision Method. The acquired full-life vibration energy spectrum of the gearbox and stress dataset are shown in Figures 4 and 5, respectively. The average amplitude of every 10000 points was calculated to reflect the bearing's degradation, and the waveform of vibration and stress signals after processing is shown in Figure 6. According to the commonly used "light-medium-heavy" classification pattern of port load, we set $k=3$. It can be seen from the smoothed vibration energy spectrum shown in Figure 7 that the bearing degradation can be divided into three stages and RUL prediction will start from the failure stage. The threshold is predetermined as the RMS value when the port maintenance personnel detect abnormal noises.

\subsection{RUL Prediction by ARIMA-CAPF Framework}

4.3.1. Load Prediction by ARIMA Model. Before system state and RUL prediction, the ARIMA model is first adopted to predict the load and corresponding operating conditions. The one-step load prediction results are shown in Figure 8. As can be seen, the ARIMA model can accurately predict the load one step ahead, and the trend of the predicted load is the same as that of the real-time load. In order to describe the accuracy of operating condition prediction more comprehensively with different steps, we have also considered the condition prediction accuracy, which is expressed as

$$
\mathrm{CPA}=\frac{n_{\text {true }}}{n_{\text {pre }}} \times 100 \%,
$$

where $n_{\text {true }}$ is the number of accurately predicted operating conditions and $n_{\text {pre }}$ is the number of operating conditions to be predicted. And the accuracy of multistep condition prediction is shown in Table 1. From Table 1, we can conclude that the prediction accuracy decreases as the prediction steps increase but generally remains in a relatively high range. This is acceptable because when the bearing's degradation process is closer to the failure time, fewer prediction steps would be needed, which means the predicted load and corresponding operating conditions are more reliable.

4.3.2. RUL Prediction by CAPF Method. In order to demonstrate the effectiveness of our proposed CAPF method and RUL prediction in dealing with dynamic conditions, we compare it with three methods, M1, M2, and M3:

$$
\begin{aligned}
& \text { M1: } x_{t}=a_{t}\left(x_{t-\Delta t}+\eta_{t} \Delta S_{t-\Delta t}+b_{t}\right)+\omega_{t}, \\
& \text { M2: } x_{t}=a\left(x_{t-\Delta t}+\eta_{o_{t}} \Delta S_{t}+b\right)+\omega_{t}, \\
& \text { M3: } x_{t}=a_{o_{t-\Delta t}, o_{t}}\left(x_{t-\Delta t}+\eta \Delta S_{t}+b_{o_{t-\Delta t}, o_{t}}\right)+\omega_{t} .
\end{aligned}
$$

The main differences between the three methods and our method are as follows.

M1 does not take the influence of load prediction into consideration, and the change of loads is represented by that of the last time step $\Delta S_{t-\Delta t}$ instead of the predicted $\Delta S_{t}$ at time $t$. The jump coefficients and degradation rates are selected according to the operating condition at time $t-\Delta t$; M2 ignores the operating conditions' influence on the jump coefficients, while M3 does not consider the influence of operating conditions on degradation rates and only considers the influence of operating conditions on jump coefficients. Those omitted factors are set as constants.

Figure 9 shows the one-step state prediction results for the methods mentioned above. ARIMA-CAPF and M1 both cause no false alarms. M2 and M3 generate more violate fluctuations and cause more false alarms. This is because both jump coefficients and degradation rates are of vital importance when state prediction is conducted.

Furthermore, ARIMA-CAPF has more accurate performance and matches the failure point, while M1 does not generate true alarms at failure time. We can find the reason from the perspective of uncertainty management. The distributions of the predicted system states shown in Figure 10 can reflect the uncertainties of the state prediction. We can find that the predicted states by the M1 method are distributed in a wider region, which indicates large uncertainties. This is because M1 has a limited ability to manage uncertainties when its operating condition information is out-of-date. Hence, M1 cannot make accurate state prediction. On the contrary, ARIMA-CAPF utilizes the operating condition information to decrease uncertainties, resulting in a narrowly distributed region and smaller errors for state prediction.

To quantitatively evaluate the accuracy of the system state and RUL prediction results, the mean absolute error (MAE) between the predicted values and the actual values is calculated using the following equation:

$$
\mathrm{MAE}=\frac{1}{N} \sum_{1}^{N}\left|y_{t}-\overline{y_{t}}\right| \times 100 \%,
$$

where $\overline{y_{t}}$ is the predicted value at time $t$ and $y_{t}$ is the actual value at time $t$.

The prediction MAEs is shown in Table 2. It can be seen that the MAEs of the proposed ARIMA-CAPF method and 


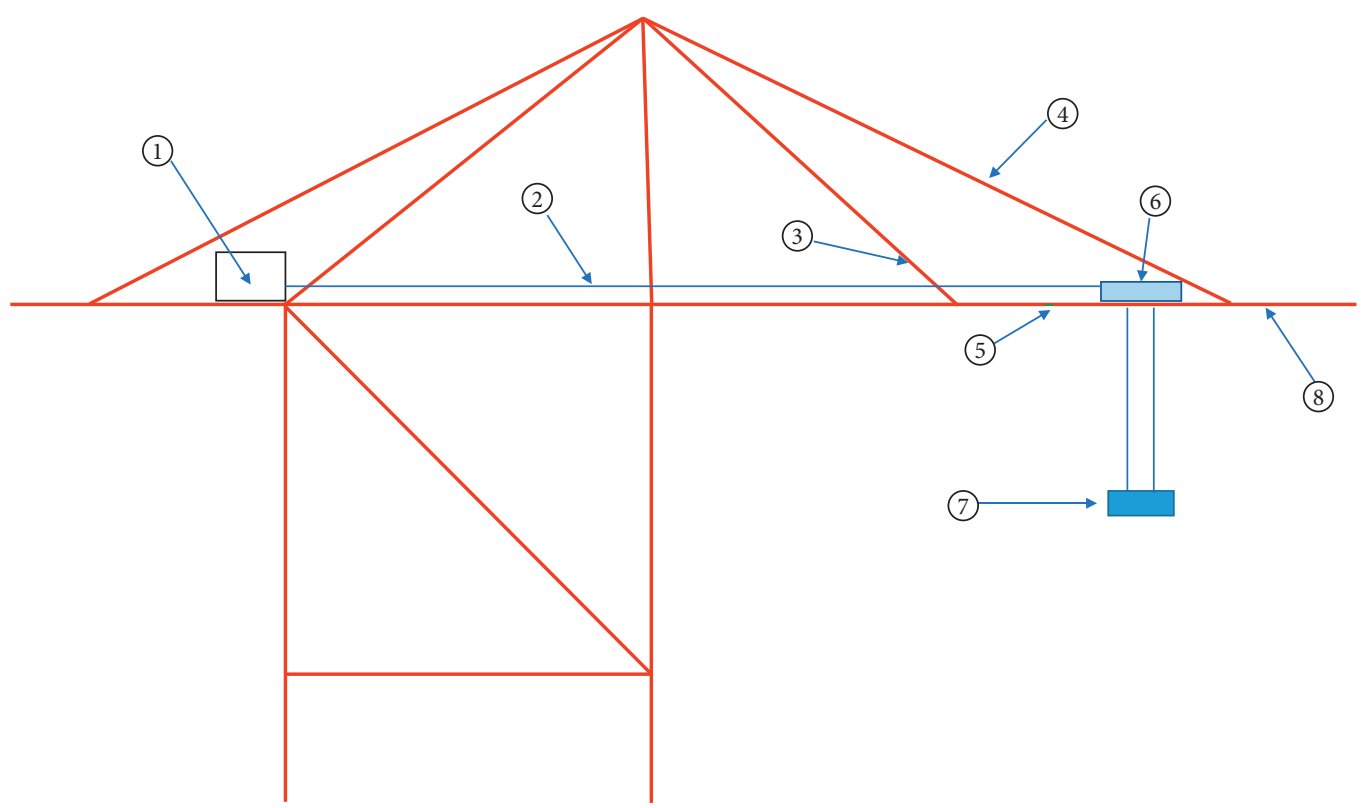

(1) Hoist Gearbox Vibration Measuring Point

(5) Stress Measuring Point

(2) Steel Rope

(6) Trolley

(3) Inner Forestay

(7) Container

(4) Outer Forestay

(8) Boom

Figure 2: Overview of QC's structure and measuring points.

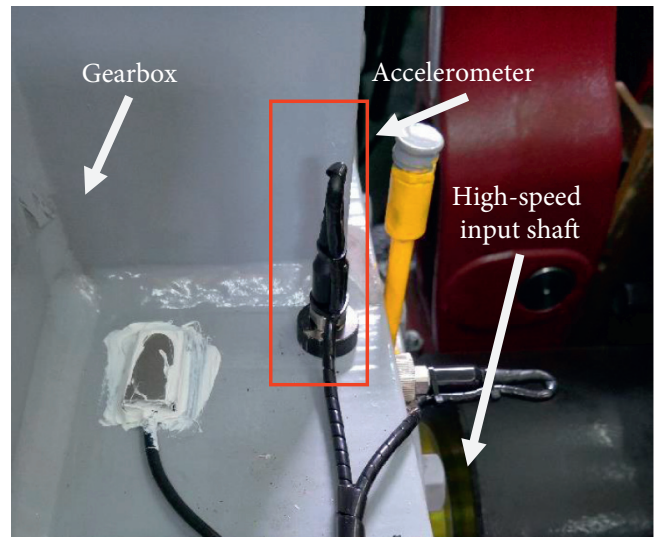

(a)

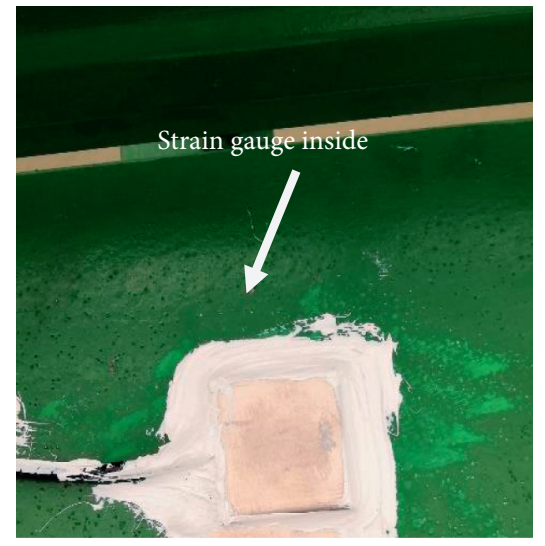

(b)

FIGURE 3: Measuring points on QC: (a) accelerometer on gearbox and (b) strain gauge on the boom.

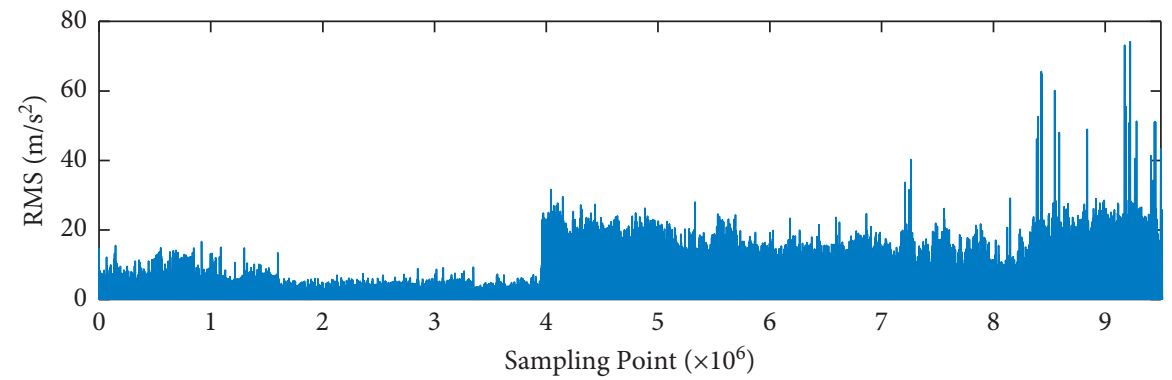

FIgURE 4: Life cycle vibration energy spectrum. 


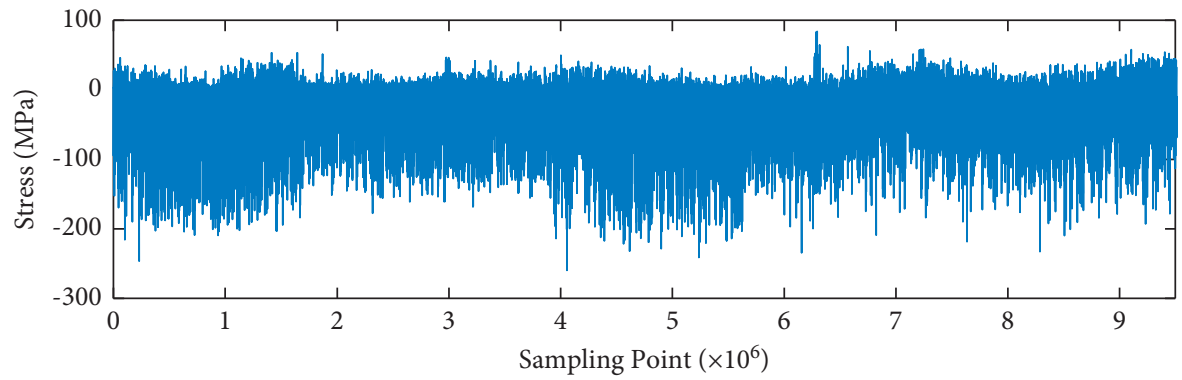

FIgURE 5: Life cycle stress signal acquired on the boom.

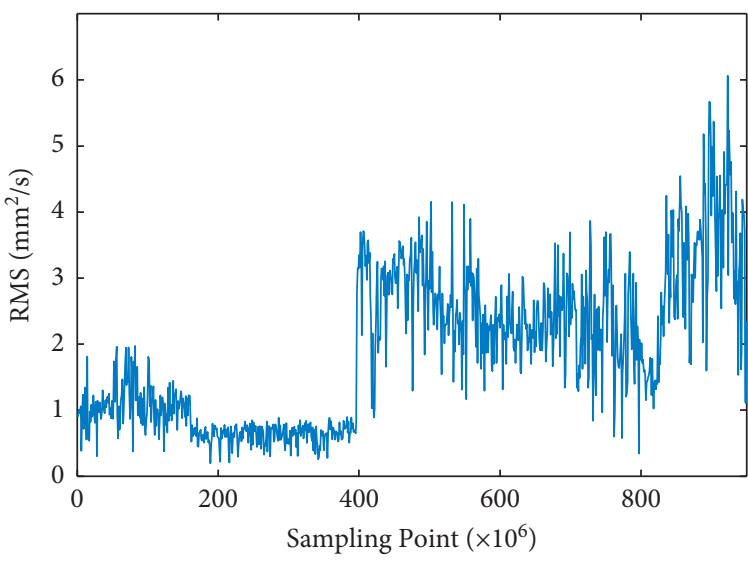

(a)

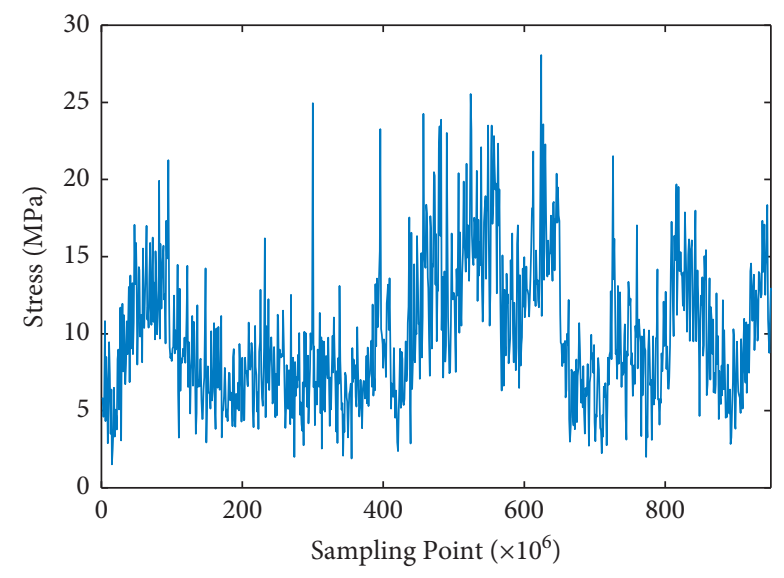

(b)

FIGURE 6: Transformed signals: (a) mean of vibration RMS and (b) mean absolute value of stress.

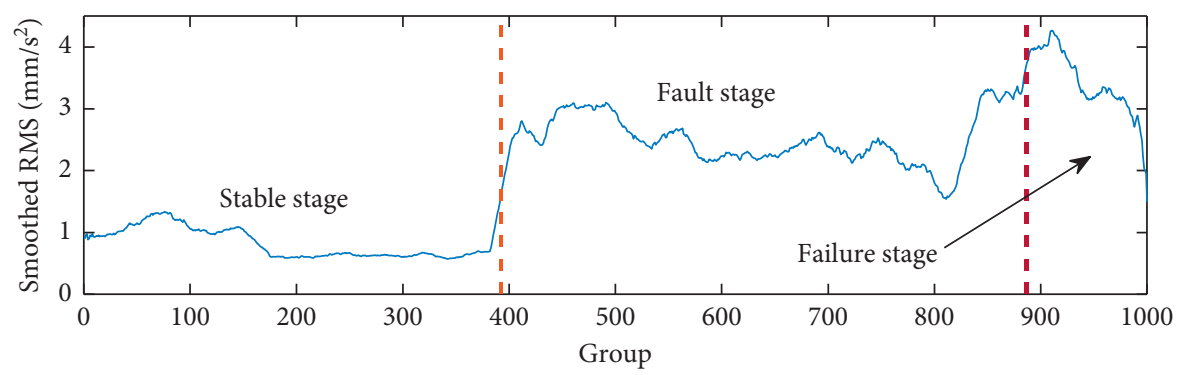

Figure 7: Stage division.

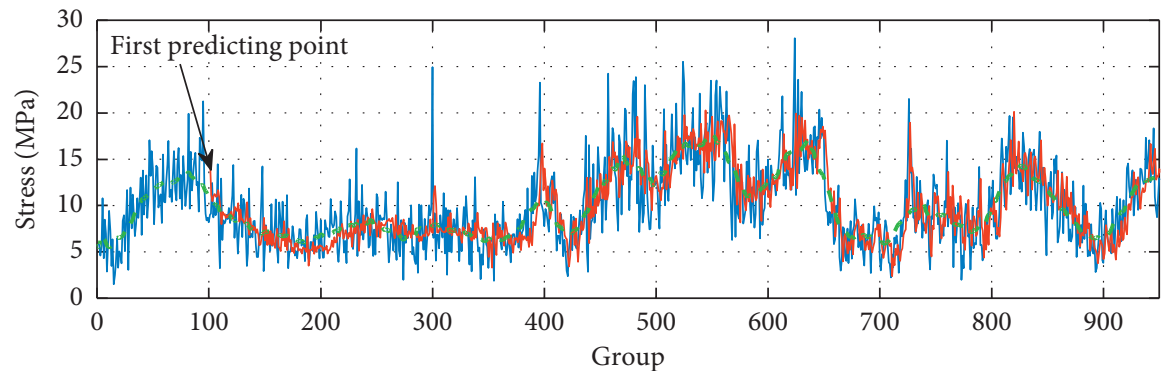

- Actual stress

_ Predicted Stress

- - - Smoothed stress

FIGURE 8: One-step stress prediction result. 
TABLE 1: Condition prediction accuracy comparison.

\begin{tabular}{lccccc}
\hline Prediction step (group) & 1 & 10 & 20 & 30 & 30 \\
\hline Condition prediction accuracy (\%) & 100 & 93.5 & 80.7 & 73.1 & 71.7 \\
\hline
\end{tabular}

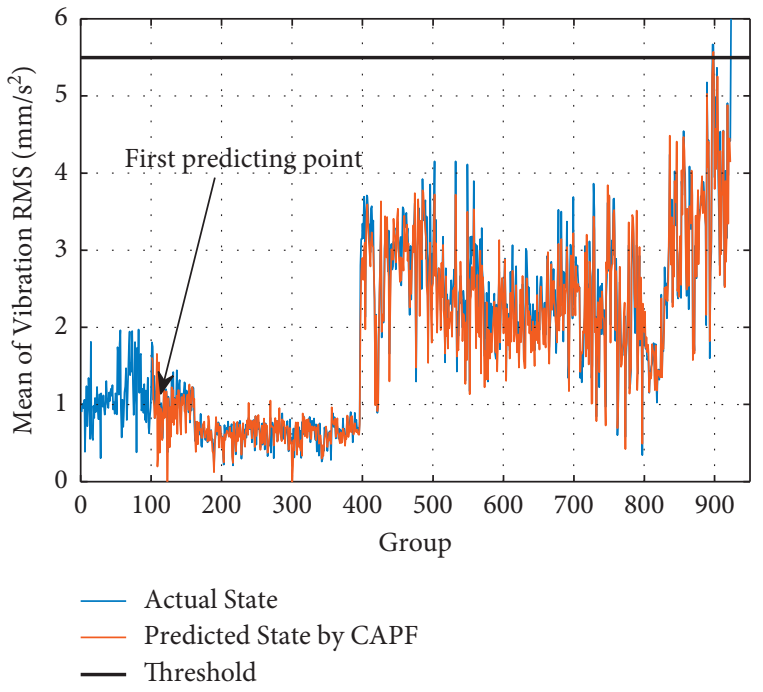

(a)

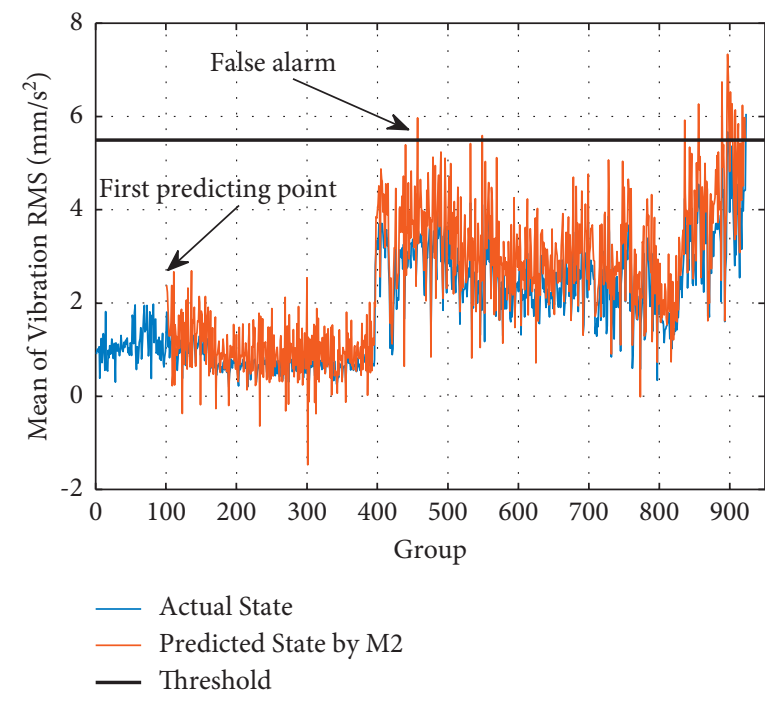

(c)

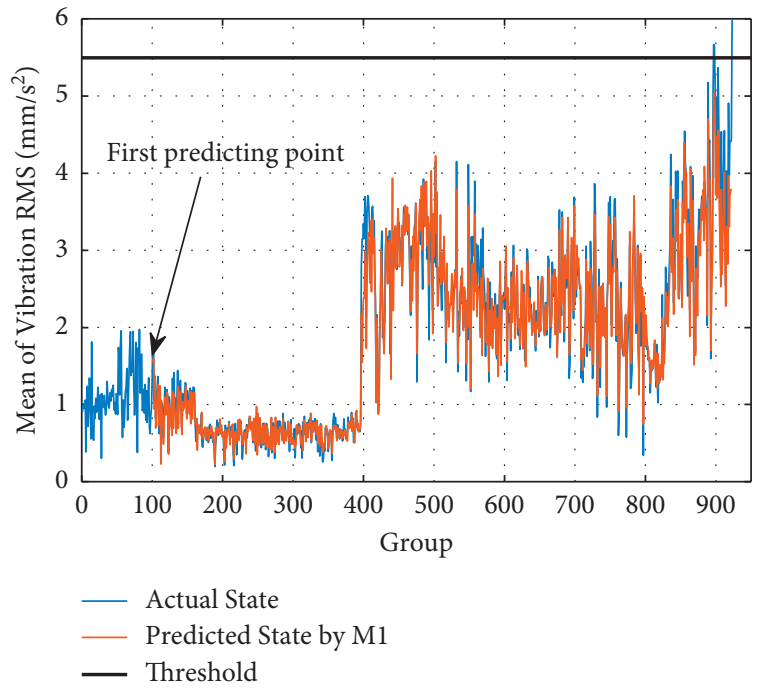

(b)

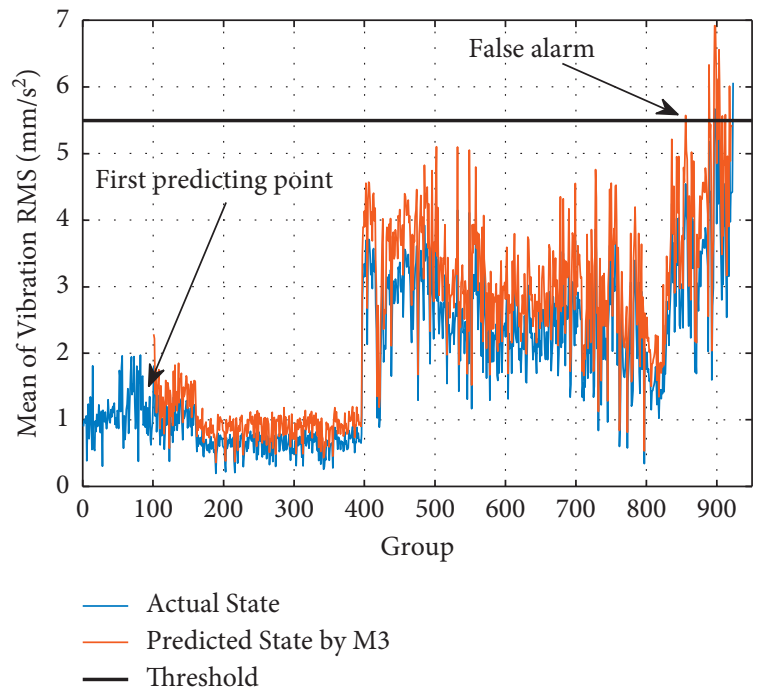

(d)

FIgURE 9: One-step state prediction results: (a) ARIMA-CAPF, (b) M1, (c) M2, and (d) M3.

M1 are both low in one-step system state prediction. But in multistep prediction, the MAE of the M1 method increases dramatically and becomes the highest in different predicting steps, according to Table 2. This is because M1 lacks the predicting information of load changes and operating conditions. With the increase of prediction step, the lack of operating condition information would cause gradually uncontrollable state prediction errors. The predicting result of M1 indicates that accurate system state prediction cannot be completed without load prediction under dynamic load conditions. M2 and M3 generate larger MAEs than ARIMACAPF in system state prediction with every prediction step.
The reason is that the two methods ignore the influence of operating conditions on jump coefficients and degradation rates, respectively. Specifically, the MAE of M2 is larger than that of M3, which indicates that the dynamic jump coefficients have a greater influence on the prediction of system state than degradation rates. This is consistent with the conclusion drawn by $\mathrm{Li}$ et al. [18].

It also can be noticed from RUL prediction results shown in Figure 11 that M1 has no ability to predict the RUL; the prediction was not accurate almost for a lifetime. This is because when it comes to multistep prediction, the load information only relies on that of the current time and 


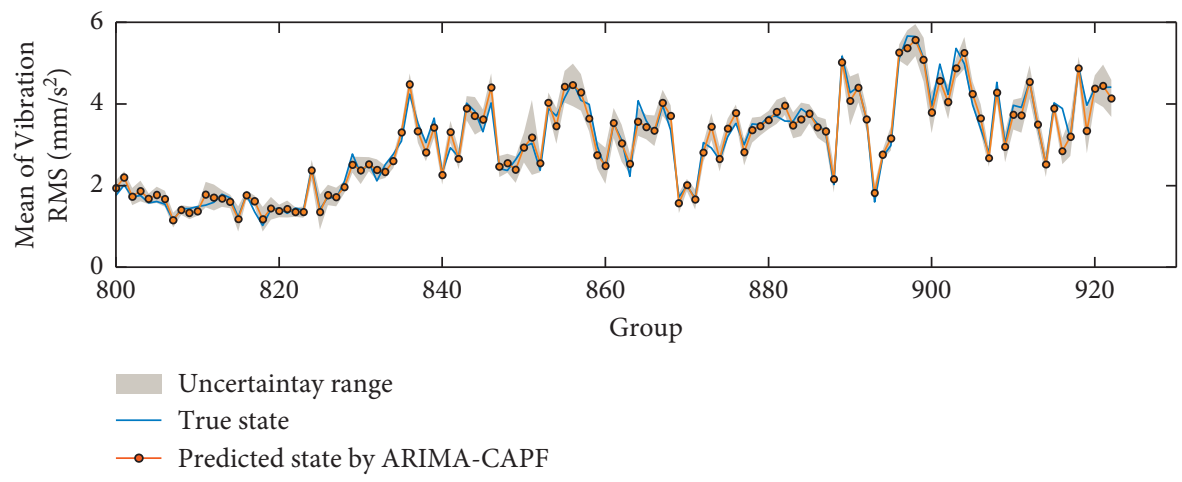

(a)

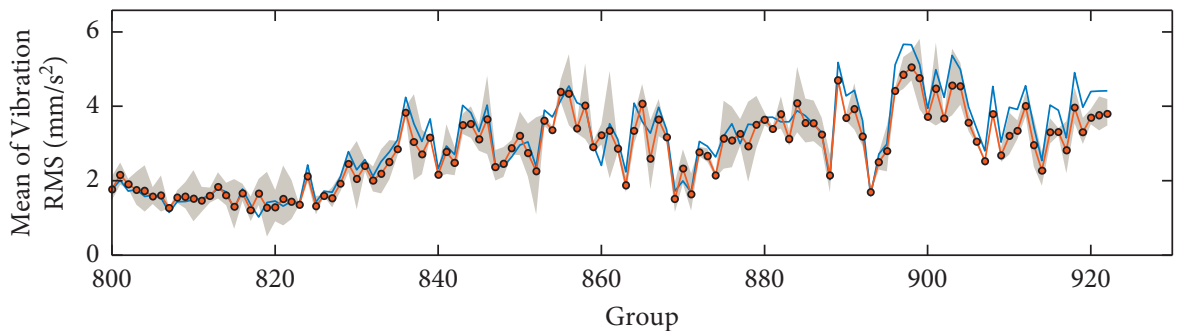

Uncertaintay range

- True state

$\multimap$ Predicted state by ARIMA-CAPF

(b)

FIgURE 10: Comparison of state prediction uncertainties.

TABLE 2: State prediction MAEs of four methods.

\begin{tabular}{lccccc}
\hline Predict time step (group) & Method & 1 & 10 & 20 & 30 \\
\hline & ARIMA-CAPF & 0.37 & 0.42 & 1.08 & 1.50 \\
MAE (\%) & M1 & 0.36 & 1.93 & 3.66 & 2.84 \\
& M2 & 0.96 & 1.77 & 3.56 \\
& M3 & 0.72 & 1.53 & 2.10 & 2.44 \\
\hline
\end{tabular}

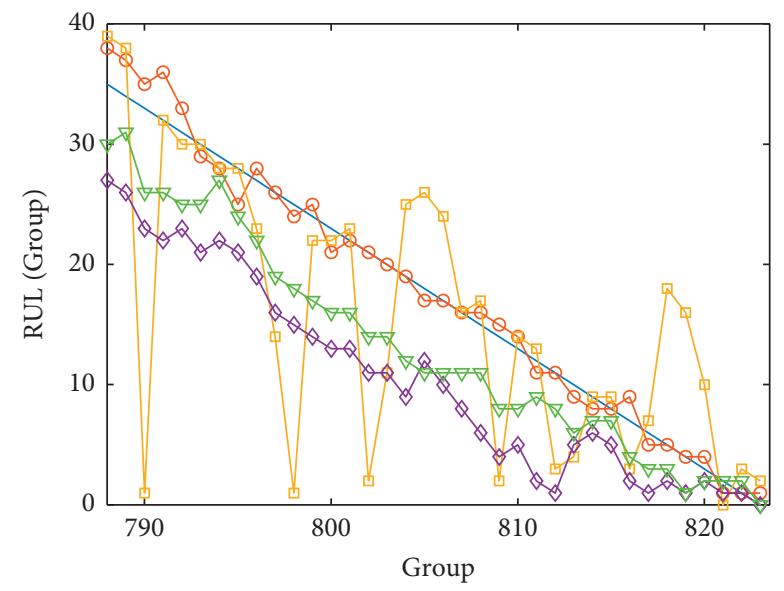

— Actual RUL

- RUL predicted by ARIMA-CAPF

$\rightarrow$ RUL predicted by M1

$\neg$ RUL predicted by M2

$\rightarrow$ RUL predicted by M3

FIGURE 11: Comparison of RUL prediction results. 
TABLE 3: RUL prediction MAEs of four methods.

\begin{tabular}{lcccc}
\hline Method & ARIMA-CAPF & M1 & M2 & M3 \\
\hline MAE (\%) & 1.0278 & 7.8056 & 6.944 & 4.3333 \\
\hline
\end{tabular}

cannot be updated further. Furthermore, it is impossible to predict RUL without accurate estimated loads and operating conditions. Once the operating condition at the current time is set as "heavy" mode, the RUL prediction result will decrease dramatically. The prediction points abruptly dropping to the horizontal axis of the coordinates of M1 have verified this inference. This also explains why M1 generates max MAEs shown in Table 3.

The RUL prediction results derived by M2 and M3 are much lower than actual RUL, and this is mainly because the system states estimated from these two methods cause lots of false alarms. Among these two methods, the predicted states of M2 are lower than that of M3, and the MAE of M2 is higher than that of M3 presented in Table 3, which once again confirms that jump coefficients have a greater impact.

However, the proposed ARIMA-CAPF method acquires the most stable and accurate predicting result among all four methods because all factors omitted in M1, M2, and M3 have been evaluated, and therefore, the effectiveness of the ARIMA-CAPF method is verified.

\section{Conclusion}

The dynamic operating conditions bring challenges to the hoisting gearbox bearing RUL prediction. Without condition prediction, the uncertainty of degradation rates and signal jumps will lower the RUL prediction accuracy. To solve this problem, we propose a framework for RUL prediction under dynamic operating conditions. Specifically, the main technical contributions of our work are summarized as follows:

(1) The proposed RUL prediction framework aims to fill the blank of load prediction in traditional RUL prediction and improve the prediction accuracy. Under this framework, the ARIMA model is able to predict the load and can maintain relatively high prediction accuracy of operating conditions with limited prediction steps.

(2) The presented CAPF algorithm can solve the problem of parameter selection when conditions switch dynamically. By this method, the system state prediction equation can adaptively select the corresponding condition parameters and update them in real time. Therefore, the ARIMA-CAPF framework is able to obtain more accurate state prediction results with fewer false alarms and realize more accurate and stable RUL predictions.

In addition, only the stress measurement point on the forestay of the quay crane is taken as the feature of the operating conditions in this study. For a complex system, a single measuring point may not be able to fully reflect the changes of the external operating conditions. We will consider the multisensor fusion technology to predict the dynamic operating conditions and RUL in future work.

\section{Data Availability}

The data used to support the findings of this study are available from the corresponding author upon request.

\section{Conflicts of Interest}

The authors declare no conflicts of interest in preparing this article.

\section{Acknowledgments}

This research received a specific grant from the National Natural Science Foundation (62073213).

\section{References}

[1] H. Meihui, H. Xiong, D. Bing, and S. Dejian, "Degradation feature extraction for the ship-to-shore crane turning point based on Weibull distribution," Journal of Vibration and Shock, vol. 38, no. 22, pp. 198-203, 2019.

[2] R. K. Singleton, E. G. Strangas, and S. Aviyente, "The use of bearing currents and vibrations in lifetime estimation of bearings," IEEE Transactions on Industrial Informatics, vol. 13, no. 3, pp. 1301-1309, 2017.

[3] G. Tang, L. I. Jianxia, and H. U. Xiong, "Accurate approximate clustering algorithm and its application in the state monitoring of crane," Journal of Donghua University, vol. 44, no. 4, pp. 590-594, 2018.

[4] D. Chen, Y. Qin, Y. Wang, and J. Zhou, "Health indicator construction by quadratic function-based deep convolutional auto-encoder and its application into bearing RUL prediction," ISA Transactions, vol. 114, Article ID 11444, 2021.

[5] K. Liu, T. R. Ashwin, X. Hu, M. Lucu, and W. D. Widanage, "An evaluation study of different modelling techniques for calendar ageing prediction of lithium-ion batteries," Renewable and Sustainable Energy Reviews, vol. 131, Article ID 110017, 2020.

[6] Y. Lei, N. Li, S. Gontarz, J. Lin, S. Radkowski, and J. Dybala, "A model-based method for remaining useful life prediction of machinery," IEEE Transactions on Reliability, vol. 65, no. 3, pp. 1314-1326, 2016.

[7] H. Liao, W. Zhao, and H. Guo, "Predicting remaining useful life of an individual unit using proportional hazards model and logistic regression model," in Proceedings of the Annual Reliability and Maintainability Symposium, pp. 127-132, IEEE, Newport Beach, CA, USA, January 2006.

[8] Y. Qian, R. Yan, and S. Hu, "Bearing degradation evaluation using recurrence quantification analysis and Kalman filter," IEEE Transactions on Instrumentation and Measurement, vol. 63, no. 11, pp. 2599-2610, 2014.

[9] S. A. Aye and P. S. Heyns, "An integrated Gaussian process regression for prediction of remaining useful life of slow speed bearings based on acoustic emission," Mechanical Systems and Signal Processing, vol. 84, pp. 485-498, 2017.

[10] Q. Wang, K. Xu, X. Kong, and T Huai, "A linear mapping method for predicting accurately the RUL of rolling bearing," Measurement, vol. 176, Article ID 176109127, 2021.

[11] J. B Ali, B. C. Morello, F. Saidi, and F. Fnaiech, "Accurate bearing remaining useful life prediction based on Weibull 
distribution and artificial neural network," Mechanical Systems and Signal Processing, vol. 56-57, pp. 150-172, 2015.

[12] L. Bian, G. Nagi, and J. P. Kharoufeh, "Degradation modeling for real-time estimation of residual lifetimes in dynamic environments," IIE Transactions, vol. 47, no. 5SI, pp. 471-486, 2015.

[13] H. Liao and Z. Tian, "A framework for predicting the remaining useful life of a single unit under time-varying operating conditions," IIE Transactions, vol. 45, no. 9SI, pp. 964-980, 2013.

[14] F. Zhao, Z. Tian, E. Bechhoefer, and Y. Zeng, "An integrated prognostics method under time-varying operating conditions," IEEE Transactions on Reliability, vol. 64, no. 2, pp. 673-686, 2015.

[15] W. Peng, Y. F. Li, Y. J. Yang, J. Mi, and H. Z. Huang, "Bayesian degradation analysis with inverse Gaussian process models under time-varying degradation rates," IEEE Transactions on Reliability, vol. 66, no. 1, pp. 84-96, 2017.

[16] P. Kundu, A. K. Darpe, and M. S. Kulkarni, "Weibull accelerated failure time regression model for remaining useful life prediction of bearing working under multiple operating conditions," Mechanical Systems and Signal Processing, vol. 134, 2019.

[17] X. Xu, S. Tang, C. Yu, J. Xie, X. Han, and M. Ouyang, "Remaining useful life prediction of lithium-ion batteries based on wiener process under time-varying temperature condition," Reliability Engineering \& System Safety, vol. 214, Article ID 214107675, 2021.

[18] N. Li, G. Nagi, Y. Lei, L. Bian, and X. Si, "Remaining useful life prediction of machinery under time-varying operating conditions based on a two-factor state-space model," Reliability Engineering \& System Safety, vol. 186, Article ID 18688, 2019.

[19] G. E. P. Box and G. M. Jenkins, Time Series Analysis: Forecasting and Control, Holden Day, San Francisco, CA, USA, 1970.

[20] G. P Zhang, "Time series forecasting using a hybrid ARIMA and neural network model," Neurocomputing, vol. 50, Article ID 50159, 2003.

[21] F. Wu, C. Cattani, W. Song, and E. Zio, "Fractional ARIMA with an improved cuckoo search optimization for the efficient Short-term power load forecasting," Alexandria Engineering Journal, vol. 59, no. 5, pp. 3111-3118, 2020.

[22] C. M. Lee and C. N. Ko, "Short-term load forecasting using lifting scheme and ARIMA models," Expert Systems with Applications, vol. 38, no. 5, pp. 5902-5911, 2011.

[23] H. Nie, G. Liu, X. Liu, and Y. Wang, "Hybrid of ARIMA and SVMs for short-term load forecasting," Energy Procedia, vol. 16, Article ID 161455, 2012.

[24] V. G. Tran, V. Debusschere, and S. Bacha, "One week hourly electricity load forecasting using neuro-fuzzy and seasonal ARIMA models," IFAC Proceedings Volumes, vol. 45, no. 21, pp. 97-102, 2012.

[25] T. T. Han, J. X. Wang, R. G. Jiao, J. Wu, and H. Guo, "Application of ARIMA model in the mid- and long-term forecast of ozone concentration," Environmental Sciences, vol. 42, pp. 1-11, 2021.

[26] R. Jamil, "Hydroelectricity consumption forecast for Pakistan using ARIMA modeling and supply-demand analysis for the year 2030," Renewable Energy, vol. 154, pp. 1541-1610, 2020.

[27] Y. Ming, D. Lan, C. Jiang, M. Xu, S. Wang, and G. Zhu, "Hybrid condition monitoring of nonlinear mechatronic system using biogeography-based optimization particle filter and optimized extreme learning machine," ISA Transactions, vol. 21, 2021.
[28] Y. Ji, Z. Chen, Y. Shen, K. Yang, S. Wang, and K. Cui, “An RUL prediction approach for lithium-ion battery based on SADE-MESN," Applied Soft Computing, vol. 104, Article ID 104107195, 2021.

[29] L. Chen, J. An, H. Wang, H. Zhang, and S. Pan, "Remaining useful life prediction for lithium-ion battery by combining an improved particle filter with sliding-window gray model," Energy Reports, vol. 6, Article ID 62086, 2020.

[30] K. Zhang, P. Zhao, C. Sun, Y. Wang, and Z. Chen, "Remaining useful life prediction of aircraft lithium-ion batteries based on F-distribution particle filter and kernel smoothing algorithm," Chinese Journal of Aeronautics, vol. 33, no. 5, pp. 1517-1531, 2020.

[31] L. Liao, "Discovering prognostic features using genetic programming in remaining useful life prediction," IEEE Transactions on Industrial Electronics, vol. 61, no. 5, pp. 2464-2472, 2014.

[32] B. Yan, X. Ma, G. Huang, and J. Zhao, "Two-stage physicsbased Wiener process models for online RUL prediction in field vibration data," Mechanical Systems and Signal Processing, vol. 152, Article ID 152107378, 2021.

[33] Y. Li, X. Huang, P. Ding, and W. Zhao, "Wiener-based remaining useful life prediction of rolling bearings using improved Kalman filtering and adaptive modification," Measurement, vol. 182, Article ID 182109706, 2021.

[34] H. Wang, H. Liao, X. Ma, and R. Bao, "Remaining useful life prediction and optimal maintenance time determination for a single unit using isotonic regression and gamma process model," Reliability Engineering \& System Safety, vol. 210, Article ID 210107504, 2021.

[35] C. P. Lin, M. H. Ling, J. Cabrera, F. Yang, D. Y. W. Yu, and K. L. Tsui, "Prognostics for lithium-ion batteries using a twophase gamma degradation process model," Reliability Engineering \& System Safety, vol. 214, Article ID 214107797, 2021.

[36] Si Xiao-Sheng, W. Wang, C.-H. Hu, and D. H. Zhou, "Remaining useful life estimation - a review on the statistical data driven approaches," European Journal of Operational Research, vol. 213, no. 1, pp. 1-14, 2011.

[37] H. O. U. MeiHui, H. U. Xiong, B. Wang, and Z BoYi, "Healthy condition recognition for quayside container crane reducer based on Weibull and GG fuzzy clustering," Journal of Mechanical Strength, vol. 41, no. 5, pp. 1023-1028, 2019.

[38] Y. Wang, Z. Wang, S. He, and Z. Wang, "A practical chiller fault diagnosis method based on discrete Bayesian network," International Journal of Refrigeration, vol. 102, Article ID 102159, 2019.

[39] Q. Huang, J. Li, and M. Zhu, "An improved convolutional neural network with load range discretization for probabilistic load forecasting," Energy, vol. 203, Article ID 203117902, 2020.

[40] H. Akaike, "A new look at the statistical model identification," IEEE Transactions on Automatic Control, vol. 19, no. 6, pp. 716-723, 1974.

[41] N. Li, Y. Lei, J. Lin, and S. X. Ding, "An improved exponential model for predicting remaining useful life of rolling element bearings," IEEE Transactions on Industrial Electronics, vol. 62, no. 12, pp. 7762-7773, 2015.

1. Please check whether reference citation corrections have been carried out correctly. 
It is very important to confirm the author(s) last and first names in order to be displayed correctly on our website as well as in the indexing databases:

\section{Author 1}

Given Names: Zhiwei

Last Name: Sun

\section{Author 3}

Given Names: Kai

Last Name: Dong

\section{Author 2}

Given Names: Xiong

Last Name: $\mathrm{Hu}$

It is also very important for each author to provide an ORCID (Open Researcher and Contributor ID). ORCID aims to solve the name ambiguity problem in scholarly communications by creating a registry of persistent unique identifiers for individual researchers.

To register an ORCID, please go to the Account Update page (http://mts.hindawi.com/update/) in our Manuscript Tracking System and after you have logged in click on the ORCID link at the top of the page. This link will take you to the ORCID website where you will be able to create an account for yourself. Once you have done so, your new ORCID will be saved in our Manuscript Tracking System automatically. 